\title{
SHARING ON WEB 3D MODELS OF ANCIENT THEATRES. A METHODOLOGICAL WORKFLOW
}

\author{
A. Scianna a*, M. La Guardia a, M. L. Scaduto a \\ a ICAR-CNR (High Performance Computing and Networking Institute - National Research Council of Italy) at GISLab c/o DARCH \\ University of Palermo, Viale delle Scienze, Edificio 8, 90128 Palermo, Italy \\ andrea.scianna@cnr.it; marcellolaguardia87@libero.it;ml.scaduto@gmail.com
}

Commission II, WG II/6

KEY WORDS: 3D, Cultural Heritage, Ancient Theatres, WebGL, HTML5, Web-Based Visualization, Knowledge sharing, Virtual Reality

\begin{abstract}
:
In the last few years, the need to share on the Web the knowledge of Cultural Heritage $(\mathrm{CH})$ through navigable 3D models has increased. This need requires the availability of Web-based virtual reality systems and 3D WEBGIS. In order to make the information available to all stakeholders, these instruments should be powerful and at the same time very user-friendly. However, research and experiments carried out so far show that a standardized methodology doesn't exist. All this is due both to complexity and dimensions of geometric models to be published, on the one hand, and to excessive costs of hardware and software tools, on the other. In light of this background, the paper describes a methodological approach for creating 3D models of $\mathrm{CH}$, freely exportable on the Web, based on HTML5 and free and open source software. HTML5, supporting the WebGL standard, allows the exploration of 3D spatial models using most used Web browsers like Chrome, Firefox, Safari, Internet Explorer. The methodological workflow here described has been tested for the construction of a multimedia geo-spatial platform developed for three-dimensional exploration and documentation of the ancient theatres of Segesta and of Carthage, and the surrounding landscapes. The experimental application has allowed us to explore the potential and limitations of sharing on the Web of 3D CH models based on WebGL standard. Sharing capabilities could be extended defining suitable geospatial Web-services based on capabilities of HTML5 and WebGL technology.
\end{abstract}

\section{INTRODUCTION}

The definition of detailed 3D models and the development of Web-based virtual reality systems represent a significant value added for protection and promotion of Cultural Heritage $(\mathrm{CH})$. In this context, recent advances within Information and Communication Technology (ICT) sector have allowed the development of new methods and technologies for sharing and browsing via the Web of 3D models of $\mathrm{CH}$ (Guarnieri et al., 2010; Gruen, 2013; Herrmann and Pastorelli, 2014).

Particularly, new methods, procedures and technologies have been proposed for the construction of Web-based applications for interactive exploration of three-dimensional models, through the integration of geometrical and semantic information into an interface (Manferdini and Remondino, 2010). Principal aim is to provide users of a new exploration experience of $3 \mathrm{D}$ multimedia model of $\mathrm{CH}$. In this perspective, the Web represents the ideal mean for accessing information, managing documentation and dissemination of $\mathrm{CH}$ values (Di Benedetto et al, 2014). All this is supported by the developments in visual computing that have opened new possibilities about documenting $\mathrm{CH}$ through the integration of geospatial data, graphical representations, multimedia contents and semantic metadata (Meyer et al., 2002; Noh et al., 2009; Remondino and Rizzi, 2010; Scianna and Sciortino, 2013; Gomes et al. 2014; Siotto et al., 2014).

Beside the possibility of creating high-detailed models, the central question that arises is how to make these threedimensional models accessible and usable by the community (Di Benedetto et al., 2014). This last one is extensive as different types of multidisciplinary users with reduced (tourists) or high (scholars and researchers) technical knowledge are interested in $\mathrm{CH}$.

Indeed, the experience so far carried out highlights the difficulties involved into the identification of a proper methodological process. Today, on the base of technology available for Web, the definition of a more suitable method to publish detailed 3D models is possible with a reduction of costs of hardware and software tools.

For this reason, more and more frequent are experimentations of $\mathrm{CH}$ interactive 3D models on the Web using open source tools and free software (Guarnieri et al., 2010).

In light of these premises, the paper illustrates an experimental framework defined to produce $3 \mathrm{D} \mathrm{CH}$ information systems, shareable on the Web. In this case, in order to allow access to multimedia representation on the Web and high-quality visualization, a WebGL-based application has been realized. It is compliant with features of most recent Web standards and technologies also running on mobile platforms, WebGL is an emerging standard from Khronos Group for accelerated 3D graphics rendering for the Web that complements HTML5 standards (Marrin, 2011). This application allows to access directly to full $3 \mathrm{D}$ vector models, without the need to install plugins or other kinds of extensions on the clients.

In order to test the validity of the method, a platform hosting the ancient theatres of Segesta (Italy) and Carthage (Tunisia), and their neighbouring landscapes has been built (webgis.gislab.unipa.it/Hologramme).

The development of these study cases has highlighted capabilities and limitations of the proposed method for Web sharing of 3D vector heritage models. Emerging aspects are (I) synchronic and diachronic representation possibilities, (II) relationships between Level of Detail (LoD) of the model vs 
access speed to geospatial 3D information, (III) multi-resolution data integration.

The remainder of this paper is organized as follows. Section II analyses issues and advances in sharing on Web $\mathrm{CH} 3 \mathrm{D}$ models. Section III presents the ancient theatres system and, particularly, describes the principal characteristics of Segesta and Carthage Theatres, on which the platform has been experimented. The methodological workflow is then presented in section IV. Finally, section V shows the results achieved and section VI draws the conclusions and open scenarios.

\section{SHARING OF CULTURAL HERITAGE ON WEB}

Publishing on the Web the knowledge acquired or available for $\mathrm{CH}$ is an effective way to promote it. To stimulate the interest of users, this knowledge could be structured in the form of multimedia contents (texts, drawings, maps, movies, 2D or 3D models), necessary also to correctly document the life cycle and features of $\mathrm{CH}$. In this perspective, one of the best and very impressive ways to transfer knowledge on $\mathrm{CH}$ is to share on the Web navigable 3D models, integrated by semantic data and other multimedia contents. Usually, this operation requires accessing to a great amount of data, whose management could be very complex and transmission through the NET very slow. Until today, 3D models have been published on the Web using non-native Web technologies, like VRML (Fig.1 - option a) or PDF3D (Fig.1 - option c) and plugins running on Web Browsers. Beside, in the past, a method for sharing of 3D urban models based on 3D geospatial Web-services (3D WFS) (Fig.1 - option b) has been studied. This method could be applied also for sharing on the Web 3D geometrical and semantic data of CH (Scianna, 2013a). It requires the existence of a proper Webserver, able to deploy 3D WFS (Web Feature Service) and WMS (Web Map Service). Besides, accessing of geospatial information through a browser requires installing on it a JAVA applet/plugin that convert 3D GML data made available by the 3D WFS running as java servlet on the server, in a format compliant with features of the browser and loaded applet/plugins.

The benefit deriving from an infrastructure based on Web services, is the possibility to grant everyone the open access to data. This requires that the interface of the Web services is well defined and documented and guaranties that everyone can write an applet/plugin in order to transform information coming from Web services allowing visualization on internet browser. Besides, writing the models inside the geospatial database requires the availability of a dedicated graphic editor (i.e. Blender software and some scripts) that should allow storing the 3D model inside the geospatial database, according to predefined conceptual model (Scianna, 2013b).

If for the built environment there exist different kinds of conceptual models like CityGML, GIANT, on the other side the proposition of a global conceptual model for the description and the building of $\mathrm{CH}$ has not been fully defined; this last aspect is a complex issue, especially considering archaeological remains. These ones are characterized by a very fragmented structure, hard to be described both on geometric and semantic point to view, so that the definition of a standard conceptual model always applicable is a complex task. This difficulty is also due to the necessary coexistence of representations characterized by very different scales, inside the same model and the same visualization.

The complexity of the structure of $\mathrm{CH}$ models and their size can also represent a problem bordering to the big data sector.

To date, doesn't exists a global standardized model applicable to all kinds of cultural goods like BIM for new constructions
(Scianna et al., 2014), even if in the last few years some authors have been proposed extensions of BIM applicable to $\mathrm{CH}$ (Dore and Murphy, 2012).

On the basis of what said before, the proposition of simple and more suitable ways to publish on the web $3 \mathrm{D}$ models is a relevant aspect; it is necessary to study both the building structure of $\mathrm{CH}$ and the most appropriate Web compliant method for sharing geospatial 3D information, in relationship with the specific $\mathrm{CH}$ to describe (Fig. 1).

We think that a possible solution should take in account capabilities of HTML5 and related WebGL technology in order to build user-friendly, scalable and adaptable platforms (Fig.1 option d).

Indeed, the proposition of a model for sharing of $\mathrm{CH} 3 \mathrm{D}$ models should necessary take in account the best balance between the levels of structuring of the model, quality of visualization and access speed on the Web.

For these reasons, the correct definition of different LoDs (connected with the discretization of each model in subcomponents) is fundamental. Besides, the Web sharing of $\mathrm{CH}$ models requires a careful study about the system usability, regarding the ways of access, the navigation speed, the characteristics of the interface and the quality of contents.

a)

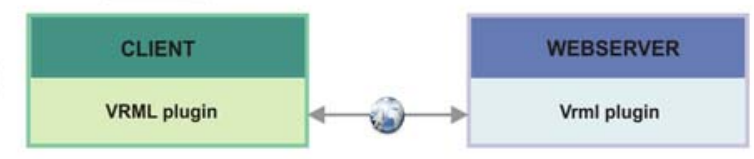

b)

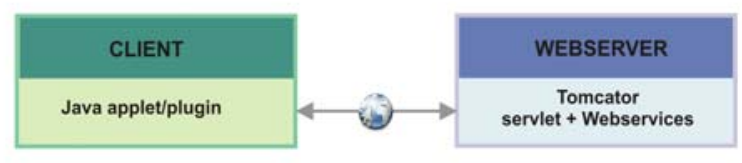

c)
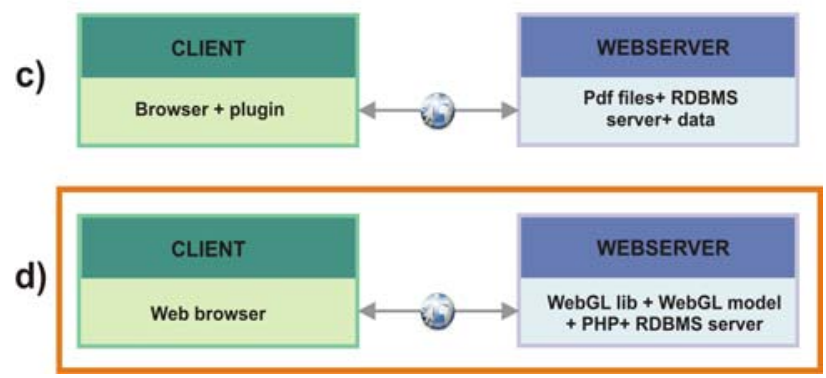

Figure 1. Different options to share and browse on Web 3D geospatial models (highlighted in orange the proposed solution).

\section{THE ANCIENT THEATRES OF SEGESTA AND CARTHAGE}

Within the experimental framework here described, the possibilities for implementing 3D $\mathrm{CH}$ information system applied to Mediterranean ancient theatres system (Segesta (Italy) and Carthage (Tunisia)) and shareable on the Web has been explored, valuing also its capabilities and limitations ( see Fig. 2).

The Theatre of Segesta (Fig. 3) dates back to the middle of the Fourth or the Second Century $\mathrm{BC}$ and it is one of the most successful examples of theatrical architecture placed in the transition from type Greek to the Roman. Although the theatre 
was located in an Elima city ${ }^{1}$, it is hypothesized that it has assumed roles and functions typical of the theatres of the Greek cities. The Theatre of Segesta, partially excavated at the beginning of the last century and recently restored, is now part of the archaeological park of Segesta and today is regularly used for theatrical performances (Tusa, 1991).

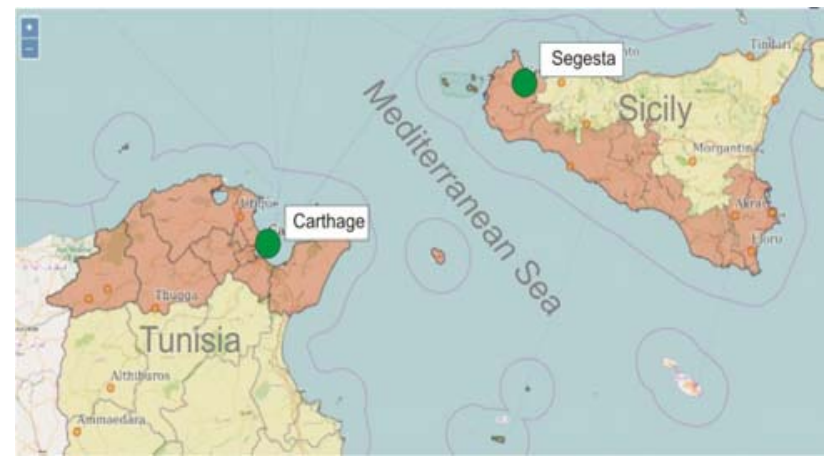

Figure 2. Localization of theatres of Segesta and Carthage (Tunisia)

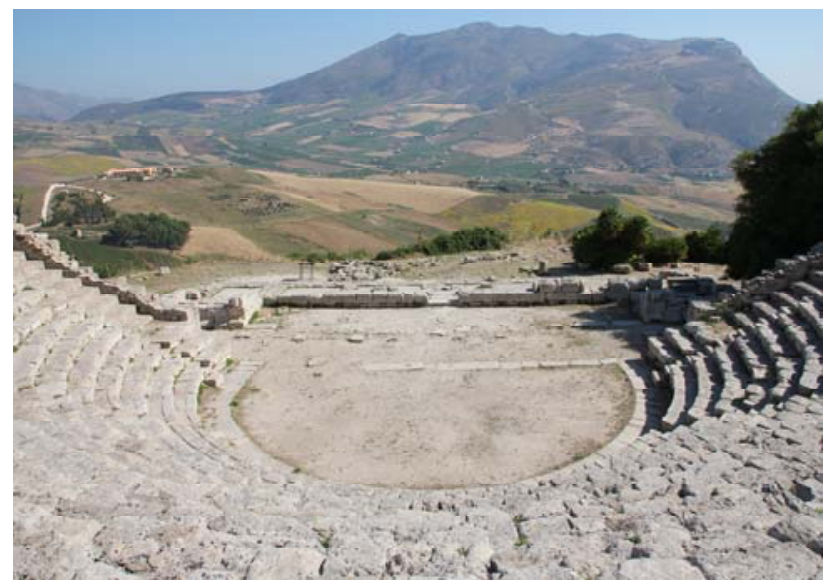

Figure 3. The Theatre of Segesta (real view)

The roman theatre of Carthage (Fig. 4), built in the Second Century BC in the north-east of the city, is an intermediate type between the Greek theatre, dug into the ground, and Roman, generally realized on an open ground. During the Fifth Century, the theatre was completely destroyed by Vandals and remained unused until the end of the Nineteenth Century. The architectural and cultural-historical importance of the Roman theatre of Carthage is related to its massive size, making it able to accommodate more than 10,000 spectators, and its peculiarities that make a real masterpiece of Roman architecture. Since 1964 the Roman theatre of Carthage welcomes the International Festival of Carthage thanks to which assumes a significant touristic, cultural, artistic and folkloristic value, of national and international level (Ros, 1996).

In both two theatres, in the last period of their life, a multistorey scena was built closing the orchestra.

1 Elimi weren't a Greek population; their origins are not yet proven, probably they arrived before Greeks in the western part of Sicily.

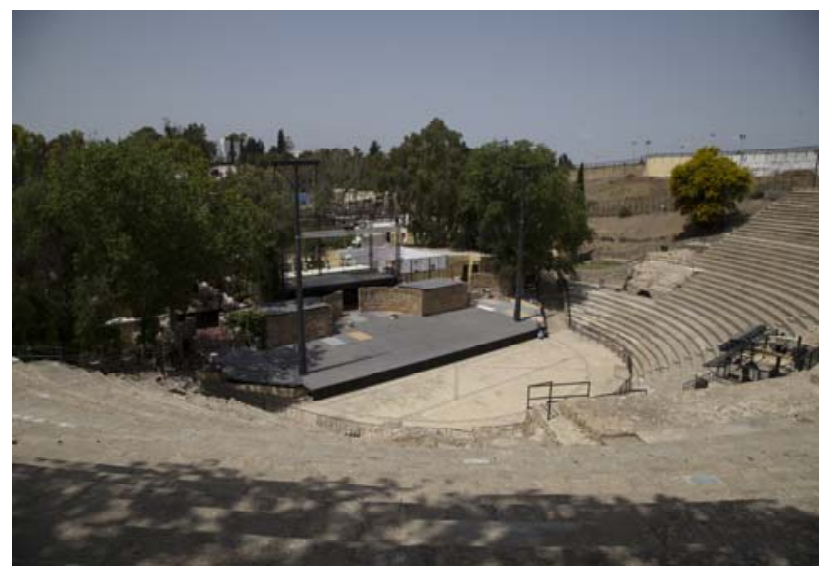

Figure 4. The Theatre of Carthage (real view)

\section{THE WORKFLOW}

The application presented here allows the observation of the $\mathrm{CH}$ (the theatres) in its original configuration and in the present. Obviously, it is possible to present $\mathrm{CH}$ also in other intermediate stages of life.

The methodological workflow for sharing on Web 3D models of ancient theatres is described in Figure 5.

Survey is the first phase. Inside it, the collection of requirements represents a step of primary importance. Requirements attain to the content of the platform, the interface and the method of exploration, formulated according to expert and visitor needs. Another important step is the acquisition of bibliographical and archival documentation required to diachronic and synchronic description of Theatres of Segesta and Carthage, as emerged from the analysis of the user's needs.

Activities carried out in subsequent phases can be synthetized in as follows:

- definition of a conceptual scheme aimed to subdivide the building into categories

- land surveys and restitutions

- 3D modelling

- construction of WebGL visualization system

- user interface construction

- browsing on internet

are detailed in the following subparagraphs.

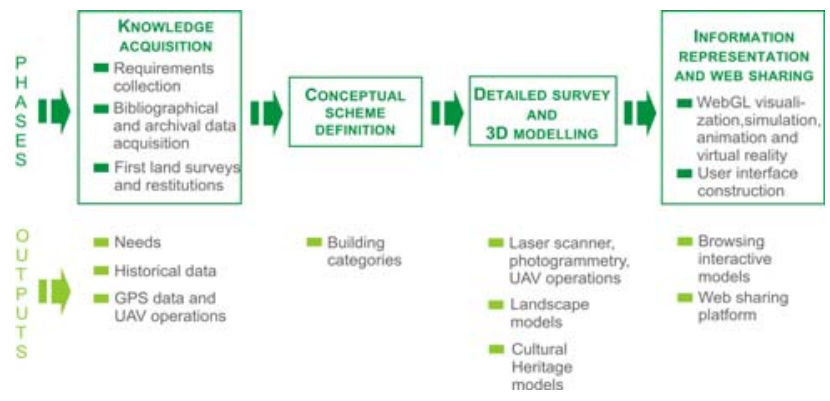

Figure 5. The methodological workflow

The global workflow, here applied to theatres, can be extended to other archaeological or historical objects. Besides WebGL technology could be applied in every other applications for sharing knowledge regarding $\mathrm{CH}$ and/or the environment.

The acquisition of historical data is necessary not only to produce information to show users but also to define correct reconstructive hypotheses to be simulated with $3 \mathrm{D}$ models. This 
phase usually requires the help of historical experts or archaeologists.

During knowledge acquisition, a global survey of the area is carried out, it is useful to acquire a rough geometric knowledge of the interested area. After the definition of conceptual schema, a detailed survey step is carried out. Survey operations must be calibrated respecting the LoDs required for construction of 3D models; generally, acquiring of data with different type of instruments is necessary, observing $\mathrm{CH}$ from different points of view and different distances. In order to guarantee the completeness of 3D models, during 3D reconstruction phase, the integration of data coming from different type of instruments is always necessary.

The definition of conceptual model depends on specific cultural object to be described. If an object or an artefact must be described without differentiating various components, the conceptual model is less important. In this case the object is structured as a geometric complex to be eventually connected to a description (linked to one external database record).

In our case, we refer to more complex models in which every object (building or monument) is composed by different parts according to a predefined level of detail requested.

\subsection{Definition of the conceptual model}

In our case, the definition of a conceptual model is mandatory and the model must fit the specific object. The conceptual model, described at chapter 4.1, has been defined for ancient theatres and their complementary parts. It (Fig. 6) allows to describe not only the theatre but also some simple services buildings like the scena. The section of the conceptual model describing the scena could be used for structuring other buildings present in an archaeological site. The architecture of the scena resembles that of a temple but with a different shape and a limited number of columns and lacking of other elements typical of temples.

3D modelling requires the discretization of buildings and surrounding environments (far and near landscape) into parts that represent architectural objects according to the requested LoD. Each building part can be connected to a block of semantic data (attributes) and must be stored inside the HTML file defining the WebGL environment.

For the definition of the conceptual model, a decomposition hypothesis of ancient theatres into its traditional functional areas (scena, cavea, analemma, etc.) has been formulated. This decomposition is necessary to cope with the complexity of geometric models and to define semantic and geometric hierarchies. Elements composing theatres are grouped into "classes" differentiating each other by function. The decomposition is defined also according to Italian Central Institute for Cataloguing and Documentation (ICCD) standard, in order to make 3D models suitable for connection to standardized semantic information system (Fig. 6).

Models of the two theatres consist of elements that are at different scales, often represented within the same scene with a different LoDs in relation with the aims of the visualization (Fig. 7) (for example, the statue of Apollo, the Theatre Carthage, and the surrounding landscape) (Fig. 8).

The different LoD of the objects depends on the central element of each single view or scene.

The virtual immersive exploration requires the representation both of the theatre and of the surrounding landscape in order to allow users to explore the relationships between these two elements (buildings and landscape) but also to analyse changes over space and time. These aspects are particularly important in the case of the Greek cities, positioned for urban and defensive reasons, in panoramic points (Fig. 9).

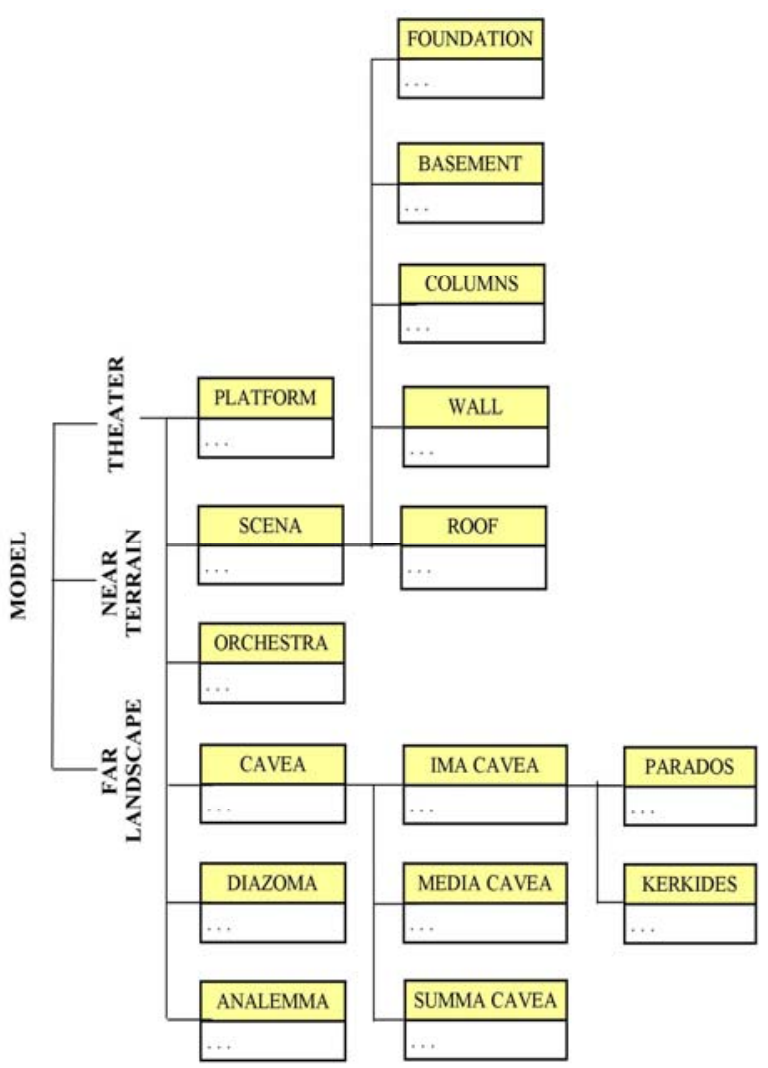

Figure 6. Structure of conceptual model

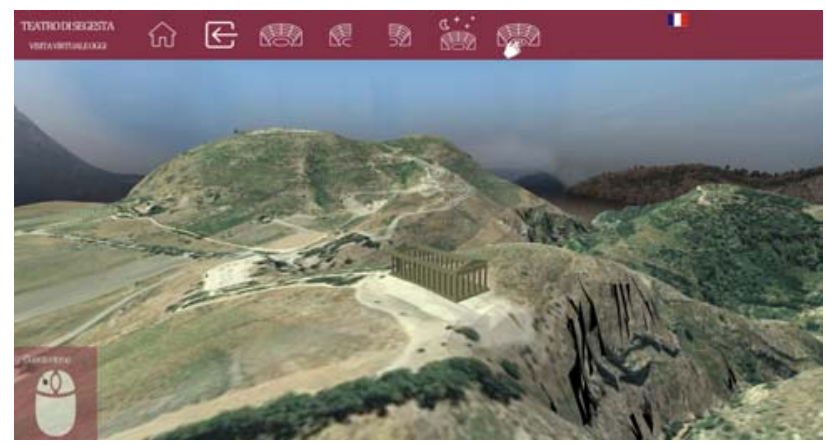

Figure 7. Archaeological park of Segesta (virtual reality view)

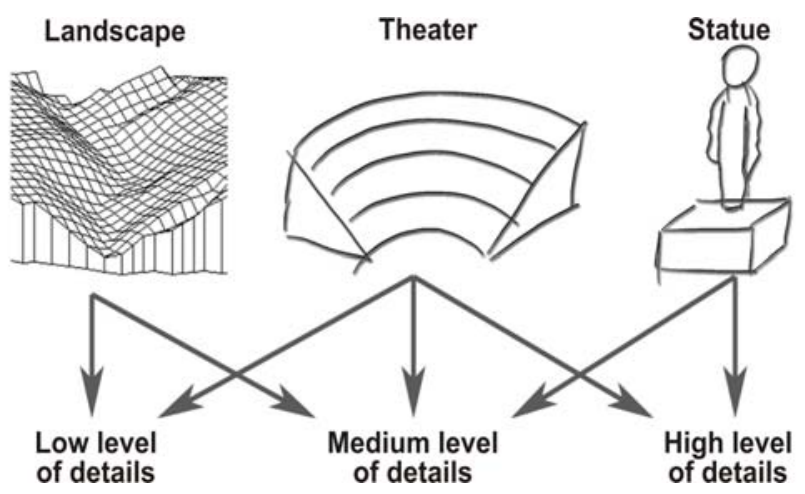

Figure 8. Platform contents at different LoD 


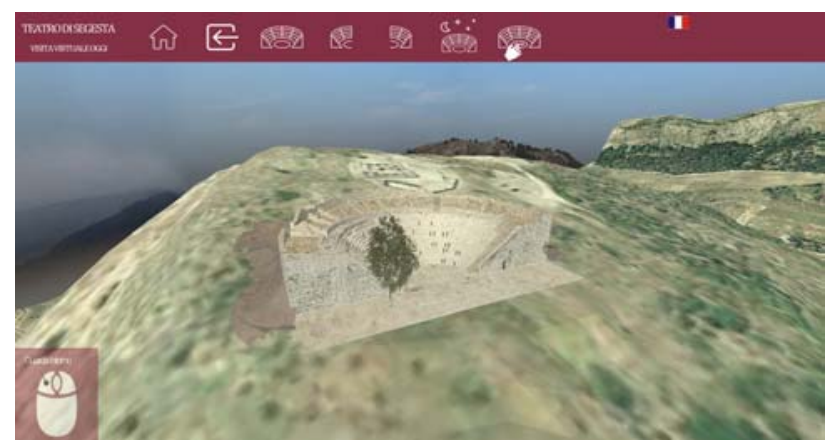

Figure 9. The Theatre of Segesta located on the top of Barbaro Mountain

\subsection{Survey and data acquisition}

The detailed survey phase represents a fundamental moment in the methodological workflow.

The construction of a multiscale model, in which theatres and surrounding areas are represented at different LoDs, requires the execution of surveys based on a combined use of different instruments (laser scanners, cameras, GPS receivers, and drones/UAVs flying at different heights). The acquisition of data with different instruments allows building of a geometric and semantics databases, where each display mode requires specific representation scales for each different objects (Fig. 10). In this perspective, the application of the most appropriate method depends on the nature of objects, as well as the purpose of their acquisition (Guarnieri et al., 2004; Pavlidis et al., 2007; Gomes et al., 2014).

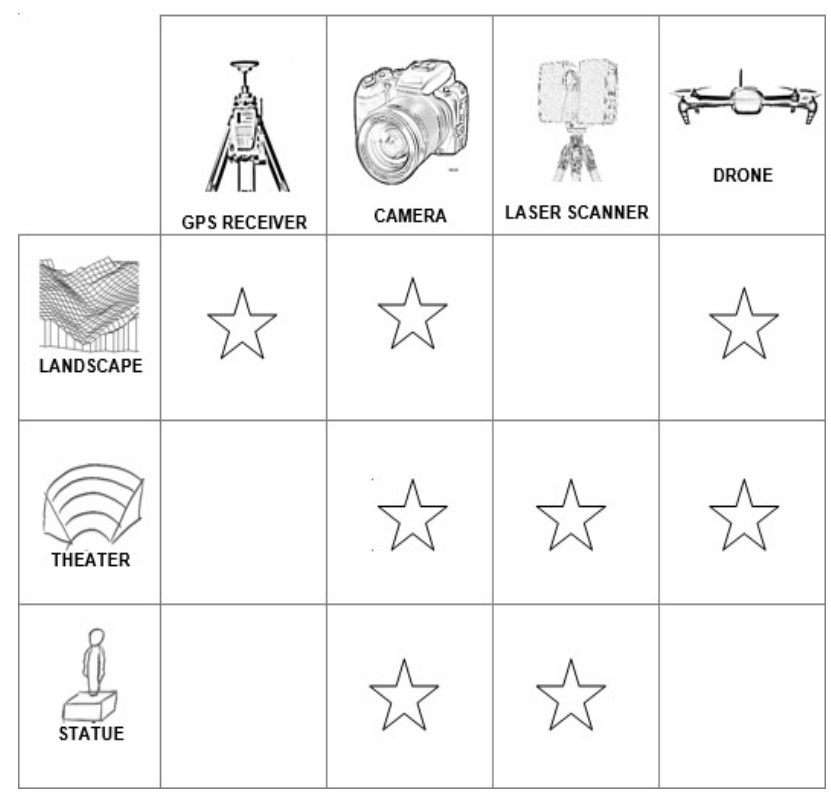

Figure 10. Different instruments suitable for survey of each element of interest

In the study cases of Segesta and Carthage, models of the territorial context were built through photogrammetry restitution, and film recording made with a flying wing, operating at height of 110 meters with 16 Megapixel calibrated camera. The orthorectification and the development of $3 \mathrm{D}$ models have been performed through some free and open source software such as Apero/MIC MAC, Visual SFM, Meshlab and Blender. 3D reconstruction was scaled and georeferred according to Ground Control Points cloud collected from GPS survey.

The model of the theatres was instead obtained by laser scanner survey, GPS survey for georeferencing and orthorectification, and multirotor survey (flying at height of 30 and 60 meters).

\subsection{D Modelling}

Main elements to be modelled are (I) landscape, distant and neighbouring; (II) theatres and annexed constructions; (III) statues.

The rough models coming out from survey must be processed in order to simplify the model and to separate objects according to the conceptual model previously defined and to needs of different kinds of visualization.

For the decimation, functions available on Meshlab and Blender software have been used in order to eliminate clusters and outliers without losing reference points for construction of meshes compliant to LoDs required for objects to be modelled.

Distant landscape is built adapting panoramic photos of the area to the internal surface of a sphere enveloping the model with its centre coincident with the centre of the model.

In order to model the near landscape, a photogrammetric reconstruction based on aerial photos taken by the fixed wing UAV has been performed; it has hallowed to obtain a point cloud of the entire affected area. Multirotors and fixed wings were used respectively for stationary and dynamic survey. Multirotors are more adapt for surveying single artefacts and areas of reduced extension, while fixed wings are more useful for larger areas.

Central moment in the modelling phase is the construction of the mesh, which consists in a three-dimensional model, originating from the two point clouds, the theatre and the ground closest to the theatre.

The reduction of the weight of the model requires a series of iterative steps to find a balanced solution concerning both the model definition, and the number of elements composing each mesh. The simplification of 3D mesh produces a reduction of its weight from $2 \mathrm{~GB}$ to $25 \mathrm{MB}$; sometimes, is necessary modelling new parts from the scratch, using the mesh coming out from surveys only as a reference. In the work of simplification, initially all the triangles that are to be almost coplanar are grouped together. This aspect is of central importance for exporting .obj object in the WebGL environment.

Geometric models are imported inside the WebGL environment using .obj formats. Actually, each model is defined using three files: (I) .obj, (II) .mtl and (III) .jpg.

The .obj file contains the definition of the mesh, which lists all the vertices with their coordinates in $3 \mathrm{D}$ space. For this reason, it is the heaviest file and it strongly influences the loading time of HTML pages. In addition, texture coordinates are associated to coordinates of vertices, always written in the same file.

The .mtl file contains all the parameters related to surfaces of the mesh, giving the possibility to change the lighting, reflection, opacity and colour of surfaces. Inside, this file the texture associated with the model is also declared.

Finally, the texture associated with the model is stored as a .jpg file. It regards the whole model and must be defined with dimension in pixels according to the power of two $(256 \times 256$, $512 \times 512,1024 \times 1024,2048 \times 2048$, etc.). The resolution of .jpg textures must be necessarily changed in order to be displayed correctly in the WebGL environment. This change does not results in distortion of the displayed image, because the pixel positions are related to the coordinates of the model in .obj files. 
In the test platform of this research, project attributes are stored inside a javascript module that encapsulates WebGL models. Functions necessary to extract data (the attributes of each building part) from a database like PostgreSQL are to be developed.

\subsection{WebGL visualization}

Main software components of HTML5/WebGL visualization system are Apache server, Three.js libraries and HTML files (inside which are contained all Javascripts).

Three.js library is a comprehensive and powerful Javascript library for creating simple 3D environments as well as interactive 3D games. Three.js library represents the core of the WebGL system, which is realized in Javascript and that allows visualizing 3D environments, calling and setting many functions like controls, camera, scene, loader, etc. (Fig. 11).

Actually, Three.min.js library has been used. It has same functionalities of Three.js library, but the .min library has all unnecessary characters removed in order to make the file size smaller so to preserve band width. In our case, three.min.js is used as builder file, OrbitControls.js for navigating inside the model, and OBJMTLLoader.js for loading 3D model.

The folders containing Three.js libraries and .HTML pages are located inside Apache HTML folder.

The system incorporates also Jquery.js library useful to simplify the client-side scripting of HTML.

The main .html file of each model is the container of Javascripts from which Three.js functions are called. In some sections of HTML pages, some variables allowing change of parameters of visualization environment, like the view point, lights, position and scale of the 3D model loaded inside, etc., are present.

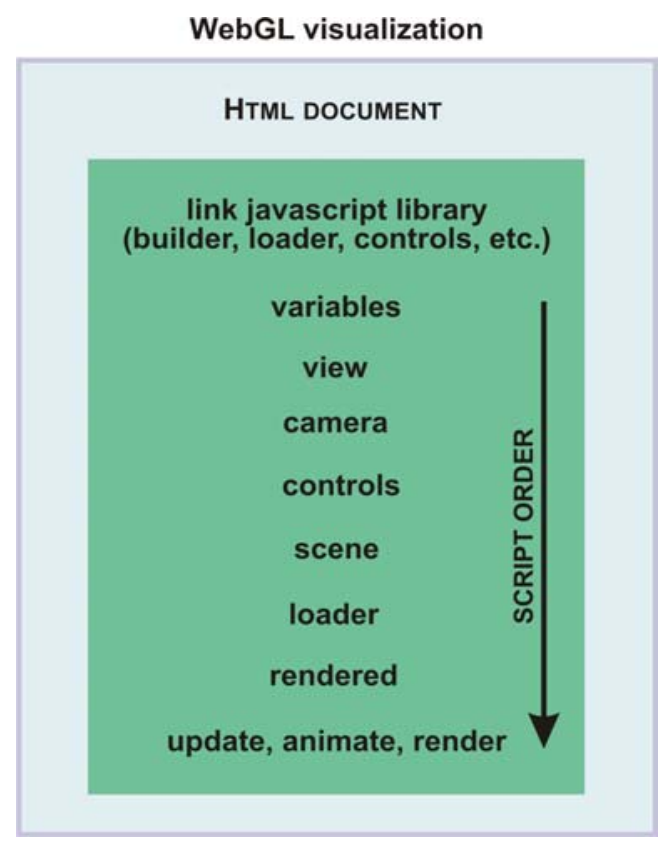

Figure 11. Structure of main HTML page of WebGL visualization environment

However there are others few parameters that cannot be changed in the .html file, but can be modified only inside the .js core files of Three.js libraries. For example, the value of the centre of rotation of each model can be changed only inside the OrbitControls.js file.
Into the HTML file is possible to insert directly the far landscape and the 3D model. The far landscape consists in a big sphere that incorporates the 3D loaded model, and the lights of the environment. On the inner surface of the sphere is projected a $360^{\circ}$ panoramic image of the site, in order to create an effective and at the same time an impressing representation of the far landscape (Fig. 12).

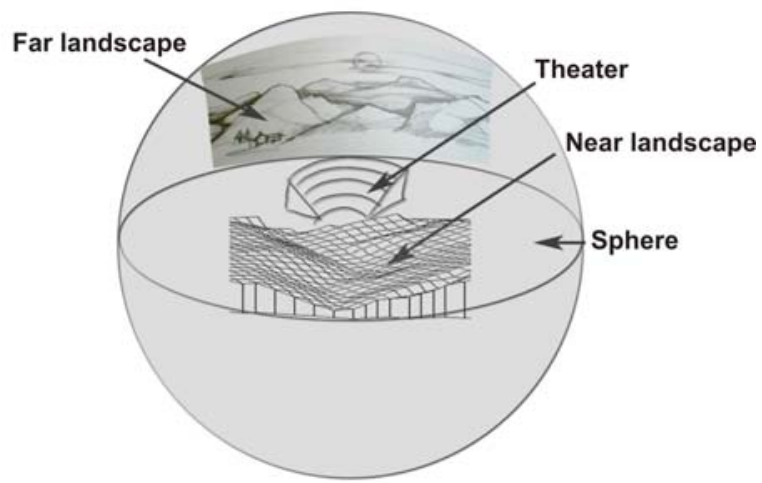

Figure 12. Structure of 3D environment

The 3D model, imported from Blender software, represents the main part of the environment. Particularly, the 3D model is composed by the theatre and the terrain close to it.

\subsection{User interface construction}

The introductory page of the platform contains a map of the Mediterranean area. In this map, there are links to the pages regarding ancient theatres of Sicily and Tunisia.

Clicking on each geographic link, the system shows the main page of the corresponding theatre. In this page is possible to acquire knowledge about the history of the theatre and its architectural features, as well as to start interactive navigation of 3D models, today and in the past (Fig. 13).

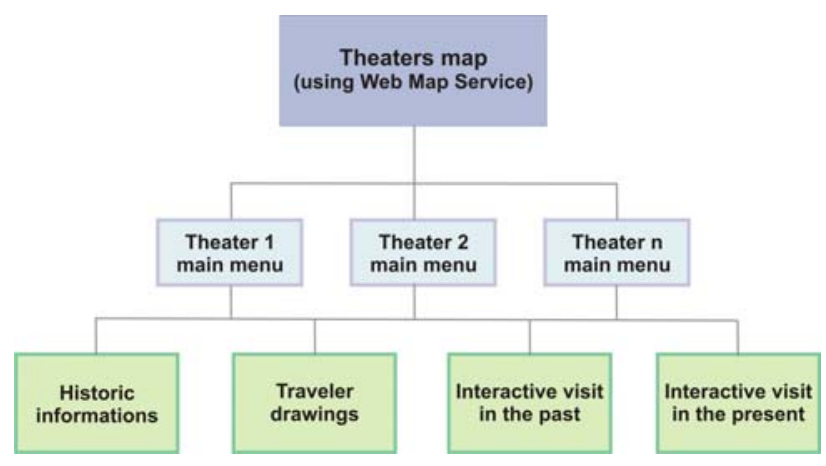

Figure 13. Structure of online platform

The interface of the interactive navigation system is created in order to give users the possibility of different kinds of navigation, using different interfaces, in order to make the experience as interesting as possible.

For every type of interface, there is a command description, in order to make the model more user-friendly. Moreover, the command controls for movement are very simple (Fig. 14).

The navigation inside the model takes place using mouse buttons or arrow keys of the keyboard. 


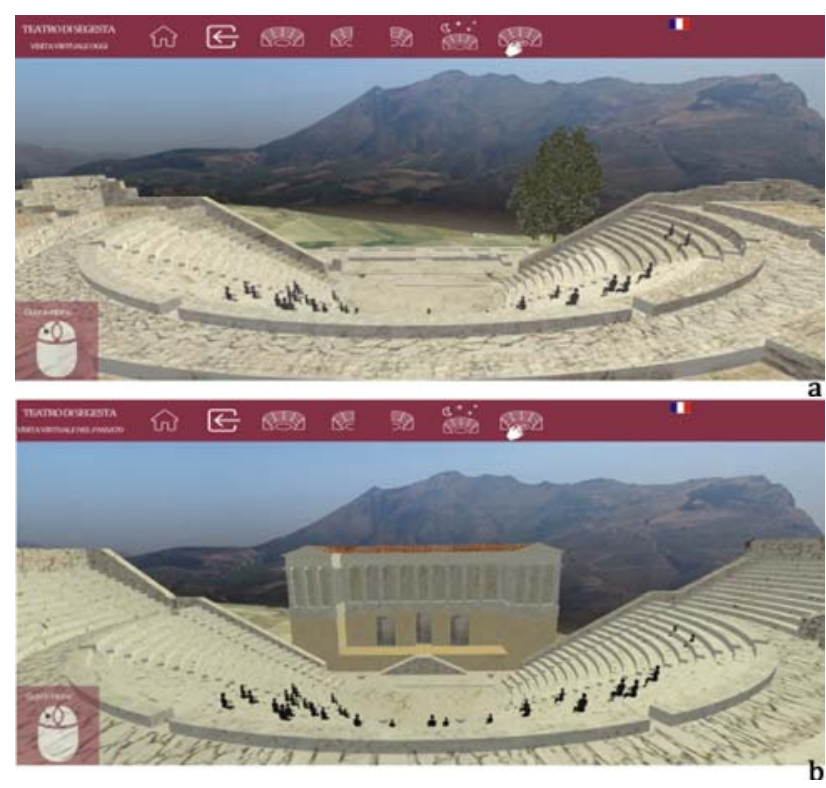

Figure 14. Interactive free navigation inside the Segesta Theatre today (a) and in the past (b)

\section{RESULTS}

The research work above described represents one of first examples of sharing on Web 3D geospatial data of archaeological Mediterranean theatres. It has been demonstrated that it is possible sharing on Web complex $\mathrm{CH} 3 \mathrm{D}$ models using simplified methods compliant with present Web visualization HTML5 and WebGL technologies and tools.

Particularly, the experimentation here presented has allowed (I) to produce and test an environment for networking $\mathrm{CH}$ geospatial information that can be shared without use of additional plug-ins or applets, and in toto based on free and open source solutions; (II) to share, view and browse interactively ancient theatre 3D models; (III) to realize ancient theatres 3D models contextualized in their environment and surrounding landscape; (IV) to handle fully 3D multi-resolution datasets; (V) to start reflections about the right balance between LoD of the model and speed access to geospatial 3D information. This latter aspect is strictly linked to the typology of $\mathrm{CH}$ objects to model, to environmental context within they are inserted and to representation scale. The downloading time of .obj models depends on their size; the size of the texture has less influence on downloading time, while has great importance for the visualization of models on mobile devices.

In this regard we can say that the system here presented has a good level of scalability. The navigation can be performed with different kinds of devices like desktop PCs, Tablets and smartphones.

Hardware limitations regard only ram of mobile devices (requiring a minimum of $2 \mathrm{~GB}$ ) and compatibility with WebGL implementation (that derives from the availability of OpenGL supported hardware).

On the software side, almost all web browsers are HTML5 and WebGL compliant and 3D models are navigable using all operative systems like Windows, Linux, Mac and Android.

Besides, a reduction of textures' resolution (related to the reduction of screen resolution) is necessary to extend the platform from desktop to mobile devices.

In addition, the research work presented here is useful as experimental model for future developments related to the networking of 3D models of $\mathrm{CH}$ via Web services.

\section{CONCLUSIONS AND OPEN SCENARIOS}

As the current literature demonstrates, the issue of sharing on Web CH 3D models represents an interesting aspect that is still all in a dynamic state of development (Agugiaro et al., 2011; Scopigno et al., 2011). Indeed, although the scientific community has been working on the $3 \mathrm{D}$ representation of individual CH (Remondino and Rizzi, 2010; Gomes et al., 2014), little has been done with respect to the modelling of archaeological heritage emergencies inserted on its landscape. Therefore, although the results of this study are partial, as the research activity is in progress, this work would highlight how it is possible create $\mathrm{CH} 3 \mathrm{D}$ models, shared on the Web, entirely based on open source and on the characteristics of HTML5.

WebGL is a recent technology (Marrin, 2011) not fully adopted in every internet browser, but is relevant because can be natively implemented inside browsers well supported by HTML5.

Furthermore, unlike the systems based on oblique photogrammetry, the system herein illustrated employs a full $3 \mathrm{D}$ models that can allow the distinction of individual parts of each model to connect to the semantic information so to realize a full 3D Geospatial Information System.

The tested experimental 3D $\mathrm{CH}$ information system allows getting simple information about the objects that compose it while browsing. Its structure allows to connect the components of the model to a geospatial database in order to obtain extended semantic information.

At current state of development, with the assistance of a webmaster, other 3D models can be uploaded on the WebGL application; the author of the model must respect some requirements:

- 3D CH models must be converted in .obj format;

- every uploaded model must have a mandatory size limited to 10-15 MB. Also for this reason is necessary to divide the entire model in smaller components.

- the texture associated to the .obj model, in order to be visualized, needs a pixel size equal to the power of two (the dimension of the texture can range from 1024 to 4096, depending on extension of the surface of $3 \mathrm{D}$ objects ), that must be correctly wrapped before.

- in the case of platforms for mobile devices, the texture must have a maximum dimension of $1024 \times 1024$ (lower is better), and must be exported in .jpg extension.

To be fully compliant to WEB 3.0 principles, these models should be explored, modified and uploadable by users in a predefined platform. This would be possible recurring to definition of 3D geospatial Webservices allowing full sharing of 3D models, making use of graphic information structured according to WebGL standards.

Despite this being the ultimate goal of our research project, at the present stage the developed framework allows exploring models and uploading new ones with similar characteristics; operatively, uploading of models requires today the assistance of Webmaster of the platform. Anyway this is still innovative for $\mathrm{CH}$ sector in which multimedia 3D information systems showing $\mathrm{CH}$ objects and their surrounding environment are not enough diffused; moreover, 3D WebGL models, accessible through Web browsers, are certainly less available in respect to those one that require the use of plugins to explore them.

Besides, another issue of interest is the speed of access. In this specific case, possible solutions could be (I) the structuring of a multiscale model that uses objects with different LoDs according to different scales of visualization; (II) the implementation of streaming technologies or Web services that allow to transfer segmented information of the model to the 
browser as a function of visualization scale; (III) the division of textures of the model in quad in such a way that furthest elements prove less defined, and then with a lighter level of description; (IV) the definition of a specific format that allows further compression of data.

\section{ACKNOWLEDGEMENTS}

This research work was supported and founded by CICI association within the HOLOGRAMME Project PS 2.2.003 P.O. Italy-Tunisia 20072013 - Program ENPI of Trans-frontier Cooperation Italy-Tunisia 2007-2013.

\section{REFERENCES}

Agugiaro, G., Remondino, F., Girardi, G., von Schwerin, J., Richards-Rissetto, H., De Amicis, R., 2011. QUERYARCH3D: Querying and Visualising 3D Models of a Maya Archaeological Site in a Web-Based Interface. Geoinformatics, CTU, FCE, pp. 10-17.

Di Benedetto, M, Ponchio, F., Malomo, L., Callieri, M., Dellepiane, M., Cignoni, P., Scopigno R., 2014. Web and Mobile Visualization for Cultural Heritage. In: M. Ioannides and E. Quak (Eds.): 3D Research Challenges, LNCS 8355, Springer-Verlag Berlin Heidelberg, pp. 18-35.

Dore, C., Murphy, M., 2012. Integration of Historic BIM (HBIM) and 3D GIS for Recording and Managing Cultural Heritage Sites. In: 18th International Conference on Virtual Systems and Multimedia (VSMM) Proceedings (Milano 2012), pp. 369-376. IEEEXplore digital library (2012).

Gomes, L., Pereira Bellon, O. R., Silva, L., 2014. 3D reconstruction methods for digital preservation of cultural heritage: A survey. Pattern Recognition Letters, 50, pp. 3-14

Gruen, A., 2013. Reality-Based Virtual Models in Cultural Heritage. In: M. Falser and M. Juneja (eds.), 'Archaeologizing' Heritage?, Transcultural Research - Heidelberg Studies on Asia and Europe in a Global Context, Springer-Verlag Berlin Heidelberg.

Guarnieri, A., Vettore, A., El-Hakim S., Gonzo, L., 2004. Digital photogrammetry and laser scanning in cultural heritage survey. Int. Arch. Photogram. Remote Sensing Spatial Info. Sci., 35, pp. 154-159.

Guarnieri, A., Pirotti, F., Vettore, A., 2010. Cultural heritage interactive 3D models on the Web: An approach using open source and free software. Journal of Cultural Heritage, 11, pp. 350-353.

Herrmann, H., Pastorelli, E., 2014. Virtual Reality Visualization for Photogrammetric 3D Reconstructions of Cultural Heritage. In: L.T. De Paolis and A. Mongelli (Eds.): AVR 2014, LNCS 8853, Springer International Publishing Switzerland, pp. 283295.

Manferdini, A. M., Remondino, F., 2010. Reality-Based 3D Modeling, Segmentation and Web-Based Visualization. In: M. Ioannides (Ed.): EuroMed 2010, LNCS 6436, Springer-Verlag Berlin Heidelberg, pp. 110-124.

Marrin, C, 2011. WebGL specification. Khronos WebGL Working Group.
Meyer, E., Grussenmeyer, P., Perrin, J.P., Durand, A., Drap, P., 2002. A Web information system for the management and the dissemination of Cultural Heritage data. Journal of Cultural Heritage, 3, pp. 325-331.

Noh, Z., Shahrizal Sunar, M., Pan, Z., 2009. A Review on Augmented Reality for Virtual Heritage System. In: M. Chang et al. (Eds.): Edutainment 2009, LNCS 5670, Springer-Verlag Berlin Heidelberg, pp. 50-61.

Pavlidis, G., Koutsoudis, A., Arnaoutoglou, F., Tsioukas, V., Chamzas, C., 2007. Methods for 3D digitization of Cultural Heritage. Journal of Cultural Heritage, 8, pp. 93-98.

Remondino, F., Rizzi, A., 2010. Reality-based 3D documentation of natural and cultural heritage sites-techniques, problems, and examples. Applied Geomatics, 2, pp. 85-100.

Ros, K. E., 1996. The Roman Theater at Carthage. American Journal of Archaeology, 100(3), pp. 449-489.

Scopigno, R., Callieri, M., Cignoni, P., Corsini, M., Dellepiane, M., Ponchio, F., Ranzuglia, G., 2011. 3D models for Cultural Heritage: beyond plain visualization. IEEE Computer, 44(7), pp. 48-55.

Scianna, A., 2013a, Experimental studies for the definition of 3D geospatial Web services. Applied Geomatics, 5(1), pp. 5971.

Scianna, A., 2013b. Building 3D GIS data models using open source software. Applied Geomatics, 5(2), pp. 119-132.

Scianna, A., Sciortino, R., 2013. Utilizzo di strumenti free e open source per la fruizione di modelli 3D di siti archeologici basati sul formato PDF. Archeologia e calcolatori, 4, pp. 202208.

Scianna, A., Gristina, S., Paliaga S., 2014. Experimental BIM Applications in Archaeology: A Work-Flow. In: M. Ioannides et al. (Eds.): EuroMed 2014, LNCS 8740, Springer International Publishing Switzerland, pp. 490-498.

Siotto, E., Callieri, M., Pingi, P., Scopigno, R., Benassi, L., Parri, A., La Monica, D., Ferrara, A., 2014. From the archival documentation to standardised Web database and $3 D$ models The case study of the Camaldolese Abbey in Volterra (Italy). International Conference on Cultural Heritage and New Technologies, Vienna.

Tusa, V., 1991. Segesta. Sellerio Editore. Palermo. 